\title{
An investigation of the association between plasma linoleic acid, metabolic health and haplotypes of FADS1/2 genes
}

\author{
K. Li ${ }^{1}$, L. Brennan ${ }^{1}$, F.J. Bloomfield ${ }^{2}$, D. J. Duff ${ }^{2}$, M.J. Gibney ${ }^{1}$ and A.P. Nugent ${ }^{1}$ \\ ${ }^{1}$ UCD Institute of Food and Health, University College Dublin, Belfield, Dublin4, Ireland and ${ }^{2}$ Chemical Analysis \\ Laboratories, Dublin, Ireland
}

A recent meta-analysis of prospective cohort studies shows that dietary linoleic acid (LA) is inversely associated with CHD risk in a dose-response manner ${ }^{(1)}$. However, as an essential fatty acid, the health effects of circulating LA remain unclear. The objective of the study was to explore the association between plasma LA and markers of metabolic health, and to identify key determinants of plasma LA.

Plasma fatty acids in 820 fasting blood samples from the Irish National Adult Nutrition Survey ${ }^{(2)}$ were extracted, trans-esterified and determined using GC-MS ${ }^{(2)}$. 15 SNPs of the FADS1/2 gene were examined. Linkage disequilibrium and haplotypes were analysed using HaploView and R package Haplo.stat respectively. Haplotype effects were estimated using HapStat 3.0 ${ }^{(3)}$. Differences in demographics, dietary intakes, markers of metabolic health were compared using GLM adjusted for confounders across plasma LA quartiles using SPSS.

Concentration of biochemistry biomarkers across plasma linoleic acid quartiles in Irish adults (n=820)

\begin{tabular}{|c|c|c|c|c|c|c|c|c|c|}
\hline & \multicolumn{9}{|c|}{ Quartiles of plasma LA } \\
\hline & \multicolumn{2}{|c|}{$1(n=205)$} & \multicolumn{2}{|c|}{$2(n=205)$} & \multicolumn{2}{|c|}{$3(n=205)$} & \multicolumn{2}{|c|}{$4(n=205)$} & \multirow[b]{2}{*}{ P-trend } \\
\hline & Mean & $\mathrm{SD}$ & Mean & SD & Mean & $\mathrm{SD}$ & Mean & SD & \\
\hline \multicolumn{10}{|l|}{ Metabolism } \\
\hline Glucose $(\mathrm{mmol} / \mathrm{l})$ & $5 \cdot 71$ & $1 \cdot 21$ & $5 \cdot 23$ & 1.28 & $5 \cdot 11$ & 0.66 & 5.03 & $0 \cdot 86$ & 0.036 \\
\hline Insulin $(\mu \mathrm{IU} / \mathrm{ml})$ & 12.77 & $10 \cdot 8$ & 8.73 & $6 \cdot 15$ & $8 \cdot 97$ & $8 \cdot 08$ & 7.48 & 5.28 & $3.5 \times 10^{-5}$ \\
\hline C-Peptide (ng/ml) & 3 & $2 \cdot 51$ & 2.08 & $2 \cdot 41$ & 1.93 & 1.97 & 1.48 & 1.4 & $4.4 \times 10^{-5}$ \\
\hline Triglyceride $(\mathrm{mmol} / \mathrm{l})$ & 1.83 & 0.88 & 1.28 & 0.58 & 1.03 & $0 \cdot 38$ & 0.86 & $0 \cdot 33$ & $1.3 \times 10^{-35}$ \\
\hline HOMA-IR & 3.43 & $3 \cdot 76$ & $2 \cdot 09$ & 1.74 & $2 \cdot 16$ & 2.59 & 1.71 & 1.33 & $5.6 \times 10^{-6}$ \\
\hline QUICKI & $0 \cdot 34$ & $0 \cdot 04$ & $0 \cdot 36$ & 0.04 & $0 \cdot 36$ & 0.04 & 0.37 & 0.04 & $1.2 \times 10^{-5}$ \\
\hline \multicolumn{10}{|c|}{ Immune function and metabolic health } \\
\hline PAI-1 (ng/ml) & $25 \cdot 46$ & $9 \cdot 16$ & 24 & 9.68 & $22 \cdot 11$ & $8 \cdot 6$ & $20 \cdot 29$ & $7 \cdot 08$ & $7.2 \times 10^{-5}$ \\
\hline TNFA $(\mathrm{pg} / \mathrm{ml})$ & $7 \cdot 6$ & $2 \cdot 7$ & 6.99 & $2 \cdot 6$ & $6 \cdot 66$ & $2 \cdot 48$ & $6 \cdot 28$ & 1.55 & 0.003 \\
\hline hsCRP (mg/l) & 3.49 & $3 \cdot 27$ & $2 \cdot 84$ & 3 & $2 \cdot 35$ & $2 \cdot 4$ & $2 \cdot 08$ & $2 \cdot 09$ & 0.002 \\
\hline Adiponectin (ug/ml) & $5 \cdot 16$ & 2.69 & $5 \cdot 78$ & $3 \cdot 28$ & $6 \cdot 52$ & $3 \cdot 28$ & $6 \cdot 35$ & $3 \cdot 13$ & 0.001 \\
\hline
\end{tabular}

Comparisons of concentration of biochemical biomarkers and plasma fatty acids (log transformed for skewed valuable) across quartiles were assessed using General

Linear Contrast Model adjusted for age, body fat, blood pressure, waist circumference, waist hip ratio, smoking status, working energy expenditure and BMI.

P-values were corrected by multiple number of traits. P-values that exceed 1.0 after correction for multiple testing have been marked down to 1.000 .

Subjects in the highest quartile of plasma LA showed healthier metabolic profile in terms of blood lipids, insulin sensitivity and inflammation markers (PAI-1, hs-CRP, etc) $(P<0.001)$. The main determinants of plasma LA were waist circumference $(8 \cdot 1 \%)$, 15 -locus haplotype $(3.9 \%$ of variance), dietary PUFA intakes $(3.7 \%$ of variance) and body fat $(3.7 \%$ of variance $)(P<0.001)$. There was no haplotype $\times$ dietary PUFA interaction after adjustment for confounders including covariates, haplotype $\times$ covariate and dietary PUFA $\times$ covariate.

Adiposity, dietary PUFA intakes and haplotypes were the main determinants of plasma LA level. Higher plasma LA level was linked to a healthier metabolic profile and lower metabolic risk.

The project was funded by the Department of Agriculture, Fisheries and Food and the Health Research Board under the Food for Health Research Initiative 2007-2012. Mr Li is in receipt of a PhD student from the China Scholarship Council 2012-2016.

1. Farvid MS, Ding M, Pan A et al. Circulation (2014) 18, 1568-78.

2. Li K, Brennan L, McNulty BA et al. Mol Res Food Res (2016), DOI: 10.1002/mnfr.201500711.

3. Lin DY, Zeng D and Millikan R. Genet Epidemiol (2005), 29, 299-312. 